\title{
Laparoscopic Liver Resection
}

\author{
David A. Geller M.D., Mohammad Khreiss M.D.
}

\section{Take-Home Points}

- Laparoscopic liver resection (LLR) is safe, feasible and is rapidly gaining acceptance.

- Indications for LLR are symptomatic benign lesions, adenomas $>4 \mathrm{~cm}$, giant hepatic cysts, and well-selected hepatocellular carcinoma (HCC) or metastatic colorectal cancer (mCRC) tumors.

- Bene ${ }^{\circ}$ ts of LLR include smaller incisions, less estimated blood loss (EBL) and packed red blood cell (pRBC) transfusions, less postoperative pain, shorter length of stay, and decreased postoperative morbidity compared to open hepatic resection.

- Long-term oncologic outcomes for HCC or limited mCRC are comparable when comparing LLR to open liver resection in case-cohort matched studies.

Laparoscopic liver resection is rapidly being adopted for certain benign and malignant tumors with more than 6000 cases performed worldwide. ${ }^{1,2}$ Early reports were predominantly nonanatomic liver resections, but greater experience has led to major anatomic hepatectomies being performed laparoscopically. ${ }^{3-5}$ Surgical techniques for laparoscopic hepatectomy include pure laparoscopic, hand-assisted laparoscopic, robotic, and laparoscopic-assisted "hybrid" approaches for which the operation is started laparoscopically and completed through a small open incision. Initial fears of adverse oncologic outcomes have not been demonstrated, and many studies have reported comparable 5-year overall survival for laparoscopic hepatic resection of HCC or limited mCRC in case-cohort studies compared to open resection. ${ }^{2}$ Benefits of laparoscopic resection include smaller incisions, less EBL and pRBC transfusions, decreased narcotic requirements, shorter length of stay, and diminished postoperative morbidity compared to open hepatic resection. $^{2}$ To date, there has been no prospective randomized controlled trial published comparing laparoscopic to open hepatic resection. Two randomized clinical trials comparing laparoscopic to open liver resection for mCRC are ongoing in Europe, and hopefully they should provide important cancer survival data that eliminates the inherent selection bias of case-cohort studies.

The indications for minimally invasive hepatic resection have expanded with increased experience. Most surgeons began with peripherally located benign adenomas or symptomatic benign hemangiomas/focal nodular hyperplasia lesions and worked their way up to resection of malignant tumors, resection in cirrhotic patients with small HCC tumors, and formal anatomic resections (Table 1). The First International Consensus Conference on Laparoscopic Liver Resection was convened in $2008^{6}$ and led to the recommendations that acceptable indications for laparoscopic resections included solitary tumors $<5 \mathrm{~cm}$, preferably located in the left lateral segments 2 to 3 or in the anterior right-lobe liver segments 5 to 6. Laparoscopic left lateral sectionectomy (LLS) was identified as an operation that should be considered standard of care. Hepatic adenomas $>4 \mathrm{~cm}$ are often resected due to risk of sponatenous rupture or developing HCC. Large hepatic adenomas can resected laparoscopically if they are safely accessible in the segments mentioned above. Although some experienced hepatobiliary surgeons have reported resecting malignant tumors $>5 \mathrm{~cm}$, the general recommendations from the first International Consensus Conference on Laparoscopic Liver Resection was to limit laparoscopic resection of malignant tumors to $<$ $5 \mathrm{~cm}$.

With greater experience came the reports of laparoscopic major liver resection. ${ }^{3-5}$ An international, multicenter experience of laparoscopic major hepatectomy from six centers (three in Europe, two in the United States, one in Australia) was reported in 210 patients. ${ }^{3}$ There were 136 right and 74 left laparoscopic hepatectomies, with $54 \%$ of cases for

Abbreviations: CT, computed tomography; EBL, estimated blood loss; HCC, hepatitis C virus; LLR, laparoscopic liver resection; $L L S$, laparoscopic left lateral sectionectomy; $m C R C$, metastatic colorectal cancer; OLR, open liver resection; OS, overall survival; $P R B C$, packed red blood cell; RFA, radiofrequency From the Department of Surgery, University of Pittsburgh, Pittsburgh, PA

Potential conflict of interest: Nothing to report.

View this article online at wileyonlinelibrary.com

(c) 2014 by the American Association for the Study of Liver Diseases

doi: $10.1002 /$ cld.430 
TABLE 1 Indications/Contraindications for Laparoscopic Liver Resection

\begin{tabular}{lc}
\hline Indications & Contraindications \\
\hline Benign Liver lesions & \\
Adenomas $\geq 4 \mathrm{~cm}$ & Any contraindication to open \\
Symptomatic hemangioma & liver resection \\
Symptomatic FNH & Patients who cannot tolerate \\
Giant hepatic cysts & pneumoperitoneum \\
Indeterminant lesions & Lesions involving major \\
Malignant Liver lesions & vascular structures \\
HCC & Relative Contraindications \\
CRC metastases & Hilar cholangiocarcinoma \\
Other metastases & Multifocal bilobar mCRC \\
& ( $>4$ tumors) \\
Live donor hepatectomy & Giant tumors ( $>10 \mathrm{~cm}$ ) \\
for liver transplant(controversial) & \\
\hline
\end{tabular}

$\mathrm{FNH}$, focal nodular hyperplasia.

malignancy. Mean operating room time was 250 minutes, and there was a 14\% transfusion rate. Median tumor size was $5.4 \mathrm{~cm}$, mean margin was $11 \mathrm{~mm}$, and the R0 resection rate was $97.3 \%$, which are comparable to open resection series. Morbidity was $14 \%$, and mortality was $1 \%$ due to two deaths: one pulmonary embolus and one urosepsis. See video of removing a large, symptomatic, 14-cm left-lobe benign focal nodular hyperplasia lesion laparoscopically in a 34 year-old female (video clip 1). The European experience of 495 major laparoscopic hepatectomies across 10 centers over a 15-year period from 1996 to 2011 was recently reported. ${ }^{4}$ The authors found that $22 \%$ of all laparoscopic liver resections during the study period were for major hepatectomies, including 348 right, 108 left, and 34 trisectionectomies with a conversion rate of $11 \%$. The analysis demonstrates a variability in technique, including hepatic mobilization, parenchymal transection, and use of inflow control, which was largely center-dependent.

Another reported use of LLR is laparoscopic live donor hepatectomy for liver transplantation. This was initially reported using laparoscopic LLS for adult-to-child pediatric liver transplantation, ${ }^{7}$ and others have reported laparoscopic-assisted right-lobe donor hepatectomy. ${ }^{8}$ It is important to point out that live donor liver transplantation and the use of a minimally invasive approach for donor hepatectomy are advanced surgeries that should be performed in high volume centers of excellence with expertise in both advanced laparoscopic hepatobiliary surgery and live donor liver transplantation.

The consensus group acknowledged that indications for laparoscopic liver resection should not be expanded beyond those of open hepatic resection simply due to the availability of a minimally invasive approach. Practically, this means that small hepatic adenomas $<4 \mathrm{~cm}$ and asymptomatic benign hemangiomas or focal nodular hyperplasia lesions should not be resected and can be observed. Contraindications to laparoscopic liver resection are patients that have a contraindication to open liver resection, patients who can-
TABLE 2 Benefits of Laparoscopic Liver Resection Versus Open Liver Resection in Case-Cohort Studies

Clinical Benefits in Laparoscopic Group From 31 Case-Cohort Studies

Decreased blood loss (14 studies)

Less pRBC transfusion (4 studies)

Less pain and narcotic use ( 8 studies)

Quicker return of diet/GI function (8 studies)

Shorter length of hospital stay (24 studies)

Decreased morbidity/complications (7 studies)

GI, gastrointestinal.

not tolerate pneumoperitoneum, and patients with tumors involving major vascular structures. Relative contraindications are hilar cholangiocarcinoma, multifocal bilobar mCRC ( $>4$ tumors), and giant tumors $(>10 \mathrm{~cm}$ ) for which laparoscopic manipulation of the liver is difficult.

Many studies published using case-cohort matched patients have shown that laparoscopic hepatic resection is safe and effective and confers significant clinical benefit to the patient (Table 2). A review of 31 case-cohort matched series comparing laparoscopic to open liver resection in 1294 patients was recently published. ${ }^{2}$ Nguyen et al. found that patients undergoing laparoscopic liver resection had less blood loss, decreased narcotic requirements, quicker resumption of an oral diet, and a shorter length of hospital stay compared to the open liver resection patients. Seven of the case-cohort matched studies in this review had decreased morbidity/complications for the laparoscopic group, whereas other studies showed no difference between the groups.

Long-term oncologic outcomes comparing laparoscopic to open liver resection for HCC and mCRC have been reported. ${ }^{1-5}$ Table 3 is a summary of a case-cohort matched series comparing laparoscopic to open liver resection for HCC.

Five-year overall survival (OS) median range was 50\% to $75 \%$, and there was no difference in overall survival comparing LLR to open liver resection (OLR) in any of the reports. There were no incidences of port-site recurrences or peritoneal seeding, and some studies showed a lower incidence of postoperative liver failure or ascites in the laparoscopic group. In a meta-analysis of 15 nonrandomized, case-matched studies comparing LLR to OLR for HCC (485 LLR versus 753 OLR patients), LLR had favorable outcomes for blood loss, pRBC transfusions, postoperative morbidity, and length of stay. ${ }^{9}$ There were no significant differences in surgical margins, 5-year overall survival, or 5-year recurrence-free survival between the groups. See video of a laparoscopic right posterior hepatectomy for a 1.5-cm HCC in a 73-year old male with hepatitis $C$ virus (HCV) and Child A cirrhosis (video clip 2). The preoperative computed tomography (CT) scan (Fig. 1A) and postoperative resected specimen with negative margins (Fig. 1B) are shown. 
TABLE 3 Five-Year Overall Survival in Comparative Studies of LLR Versus OLR Liver Resection for HCC

\begin{tabular}{|c|c|c|c|c|c|c|}
\hline \multirow[b]{2}{*}{ Reference } & \multirow[b]{2}{*}{ Journal } & \multirow[b]{2}{*}{ Year } & \multirow{2}{*}{$\begin{array}{c}\text { No. Patients } \\
\text { LLR/OLR }\end{array}$} & \multicolumn{2}{|c|}{5 -Year OS \% } & \multirow[b]{2}{*}{ P Value } \\
\hline & & & & LLR & OLR & \\
\hline Kim et al. & Surg Endosc & 2014 & $29 / 29$ & 92 & 88 & NS \\
\hline Cheung et al. & Ann Surg & 2013 & $32 / 64$ & 77 & 57 & NS \\
\hline Ker et al. & Int. J Hepatol & 2011 & $116 / 208$ & 62 & 72 & NS \\
\hline Truant et al. & Surg Endosc & 2011 & $35 / 53$ & 70 & 46 & NS \\
\hline Lee et al. & W J Surg & 2011 & $33 / 50$ & 76 & 76 & NS \\
\hline Hu et al. & W J Gastro & 2011 & $30 / 30$ & 50 & 53 & NS \\
\hline Kim et al. & J Kor Surg Soc & 2011 & $26 / 29$ & 57 & 56 & NS \\
\hline Tranchart et al. & Surg Endosc & 2010 & $42 / 42$ & 60 & 47 & NS \\
\hline Sarpel et al. & $\begin{array}{l}\text { Ann Surg } \\
\text { Oncology }\end{array}$ & 2009 & $20 / 56$ & 95 & 75 & NS \\
\hline Endo et al. & $\begin{array}{l}\text { Surg Lap } \\
\quad \text { Endo Tech }\end{array}$ & 2009 & $10 / 11$ & 57 & 48 & NS \\
\hline Cai et al. & Surg Endosc & 2008 & $31 / 31$ & 50 & 51 & NS \\
\hline Kaneko et al. & Am J Surgery & 2005 & $30 / 28$ & 61 & 62 & NS \\
\hline Shimada et al. & Surg Endosc & 2001 & $17 / 38$ & 50 & 38 & NS \\
\hline
\end{tabular}

For mCRC, nine different studies of laparoscopic liver resection for limited mCRC reported 5-year OS rates from $36 \%$ to $64 \%$, with a median of $50 \%$ (Table 4 ). These 5 -year
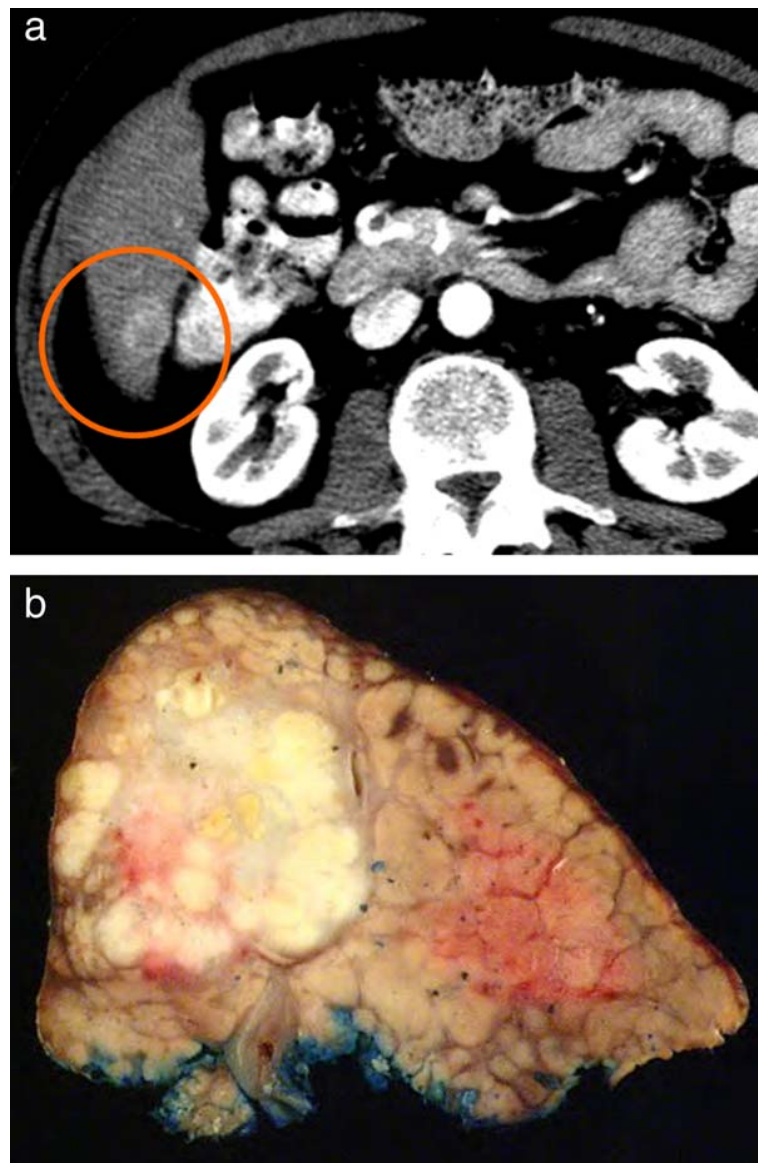

Figure 1 Laparoscopic resection of a $1.5-\mathrm{cm}$ hepatocellular carcinoma tumor in a cirrhotic patient. (1A) Preoperative CT Scan showing the hypervascualr 1.5-cm tumor in the right posterior lobe liver. (1B) Rescected surgical specimen showing a negative margin from the well-encapsulated tumor.
OS rates are comparable to open hepatic resection series. ${ }^{2,10}$ In a prospective head-to-head study from two highly specialized liver surgery centers in France, a matched comparison of 60 laparoscopic to 60 open hepatic resections for mCRC showed comparable overall- and disease-free survival rates at 1,3 , and 5 years. ${ }^{11}$ Thus, the best evidence to date does not show any adverse oncologic outcomes of laparoscopic liver resection for HCC or limited mCRC in wellselected patients.

Although many reports describe the use of radiofrequency (RFA), microwave, or ethanol ablation for small HCC tumors, hepatic resection remains the gold standard. In the setting of cirrhosis and mild portal hypertension, a minimally invasive approach for resection is preferred that decreases the disruption of collaterals to minimize postoperative liver decompensation. Ideal tumors are small $(<2 \mathrm{~cm})$ subcapsular HCC tumors in the left lateral segments or right anterior segments. Deeper tumors that are not amenable to LLR may be suitable for laparoscopic RFA, microwave ablation, or ethanol injection. If the patient is a good liver transplant candidate, then sometimes laparoscopic ablation will be offered as a bridge to transplantation rather than resection. These important decisions should be discussed at a multidisciplinary liver tumor board that includes hepatology, liver transplant surgery, and hepatobiliary surgery/surgical oncology teams.

TABLE 4 Five-Year Overall Survival of Laparoscopic Liver Resection for Metastatic Colorectal Cancer

\begin{tabular}{llccc}
\hline Reference & Journal & Year & No. Patients & 5-Year OS \\
\hline Iwahashi et al. & Surg Endosc & 2013 & 21 & $42 \%$ \\
Topal et al. & Surg Endosc & 2012 & 20 & $48 \%$ \\
Cannon et al. & Surgery & 2012 & 35 & $36 \%$ \\
Shafaee et al. & JACS & 2011 & 55 & $55 \%$ \\
Kazaryan et al. & Ann Surg & 2010 & 107 & $47 \%$ \\
Nguyen et al. & Ann Surg & 2009 & 109 & $50 \%$ \\
Castaing et al. & Ann Surg & 2009 & 60 & $64 \%$ \\
Sasaki et al. & Br J Surg & 2009 & 39 & $64 \%$ \\
Bryant et al. & Ann Surg & 2009 & 22 & $64 \%$ \\
\hline
\end{tabular}


Advances in technology with better scopes, instruments, and transection devices have greatly facilitated laparoscopic liver resection. An ongoing concern is the added cost of this technology. Cost-effectiveness was demonstrated in a study comparing laparoscopic versus open LLS in which a 50\% reduction in the hospital length of stay in the laparoscopic group more than offset the added cost of the operating room equipment. ${ }^{12}$ This cost savings was confirmed in a more recent study for hand-assisted laparoscopic hepatic resections, with financial savings of up to $\$ 11,000$. $^{13}$ Several centers have also reported use of the robot for laparoscopic liver resection. The Pittsburgh group reported 57 robotic liver resections that were case-cohort matched to 114 laparoscopic resections and showed that the use of the robot resulted in less need for a hand port, hybrid technique, or conversion to an open procedure. ${ }^{14}$ The downside was that the robot cases took longer and were not cost-effective. Further data is needed to determine the exact role of the robot in liver resection surgery.

\section{References}

1. Nguyen KT, Gamblin TC, Geller DA. World review of laparoscopic liver resection-2,804 patients. Ann Surg 2009;250:831-841.

2. Nguyen KT, Marsh JW, Tsung A, Steel JL, Gamblin TC, Geller DA. Comparative benefits of laparoscopic versus open hepatic resection: a critical appraisal. Arch Surg 2011;146:348-356.

3. Dagher I, O'Rourke N, Geller DA, et al. Laparoscopic major hepatectomy: an evolution in standard of care. Ann Surg 2009;250:856-860.

4. Dagher I, Belli G, Fantini C, et al. Laparoscopic hepatectomy for hepatocellular carcinoma: a European experience. J Am Coll Surg 2010;211:16-23.

5. Lin NC, Nitta H, Wakabayashi G. Laparoscopic major hepatectomy: a systematic literature review and comparison of 3 techniques. Ann Surg 2013;257: 205-213.

6. Buell JF, Cherqui D, Geller DA, et al. The international position on laparoscopic liver surgery: The Louisville Statement 2008. Ann Surg 2009; 250: 825-830.

7. Cherqui D, Soubrane O, Husson E, et al. Laparoscopic living donor hepatectomy for liver transplantation in children. Lancet 2002;359:392-396.

\section{Conclusion}

In conclusion, the field of laparoscopic liver resection has evolved rapidly over the past 15 years. The number of laparoscopic hepatic resections has doubled in the past 4 years from about 3000 cases worldwide through 2009 to more than 6000 cases today. Techniques have improved, and the field is reaching new horizons. To keep pace, the Second International Consensus Conference on Laparoscopic Liver Resection will be convened in October 2014 in Morioka, Japan, to develop updated consensus recommendations using an evidence-based approach.

\section{CORRESPONDENCE}

David A. Geller, M.D., Richard L. Simmons Professor of Surgery, Chief, Division of Hepatobiliary and Pancreatic Surgery, Director, UPMC Liver Cancer Center, University of Pittsburgh, 3459 Fifth Avenue, Pittsburgh, PA 15213-2582. E-mail: gellerda@upmc.edu.

8. Koffron AJ, Kung R, Baker T, et al. Laparoscopic-assisted right lobe donor hepatectomy. Am J Transplant 2006;6:2522-2525.

9. Yin Z, Fan X, Ye H, et al. Short- and long-term outcomes after laparoscopic and open hepatectomy for hepatocellular carcinoma: a global systematic review and meta-analysis. Ann Surg Oncol 2013 20:1203-1215.

10. Nguyen KT, Laurent A, Dagher I, Geller DA, et al. Minimally invasive liver resection for metastatic colorectal cancer: a multi-institutional, international report of safety, feasibility, and early outcomes. Ann Surg 2009;250:842-848.

11. Castaing D, Vibert E, Ricca L, Azoulay D, Adam R, Gayet B. Oncologic results of laparoscopic versus open hepatectomy for colorectal liver metastases in two specialized centers. Ann Surg 2009;250:849-855.

12. Vanounou T, Steel JL, Nguyen KT, Tsung A, Marsh JW, Geller DA, Gamblin TC. Comparing the clinical and economic impact of laparoscopic versus open liver resection. Ann Surg Oncol 2010;17:998-1009.

13. Cannon RM, Scoggins CR, Callender GG, et al. Financial comparison of laparoscopic versus open hepatic resection using deviation-based cost modeling. Ann Surg Oncol 2013;20:2887-2892.

14. Tsung A, Geller DA, Sukato DC, et al. Robotic versus laparoscopic hepatectomy: a matched comparison. Ann Surg 2014;259:549-555 